\title{
Thr55 phosphorylation of p21 by MPK38/ MELK ameliorates defects in glucose, lipid, and energy metabolism in diet-induced obese mice
}

\author{
Hyun-A. Seong ${ }^{1}$ and Hyunjung $\mathrm{Ha}^{1}$
}

\begin{abstract}
Murine protein serine-threonine kinase 38 (MPK38)/maternal embryonic leucine zipper kinase (MELK), an AMPactivated protein kinase (AMPK)-related kinase, has previously been shown to interact with p53 and to stimulate downstream signaling. p21, a downstream target of p53, is also known to be involved in adipocyte and obesity metabolism. However, little is known about the mechanism by which p21 mediates obesity-associated metabolic adaptation. Here, we identify MPK38 as an interacting partner of p21. p21 and MPK38 interacted through the cyclindependent kinase (CDK) binding region of p21 and the C-terminal domain of MPK38. MPK38 potentiated p21mediated apoptosis and cell cycle arrest in a kinase-dependent manner by inhibiting assembly of CDK2-cyclin E and CDK4-cyclin D complexes via induction of CDK2-p21 and CDK4-p21 complex formation and reductions in complex formation between p21 and its negative regulator mouse double minute 2 (MDM2), leading to p21 stabilization. MPK38 phosphorylated p21 at Thr55, stimulating its nuclear translocation, which resulted in greater association of p21 with peroxisome proliferator-activated receptor $\gamma$ (PPAR $\gamma$ ), preventing the PPARY transactivation required for adipogenesis. Furthermore, restoration of p21 expression by adenoviral delivery in diet-induced obese mice ameliorated obesity-induced metabolic abnormalities in a MPK38 phosphorylation-dependent manner. These results suggest that MPK38 functions as a positive regulator of p21, regulating apoptosis, cell cycle arrest, and metabolism during obesity.
\end{abstract}

\section{Introduction}

Murine protein serine-threonine kinase 38 (MPK38)/ maternal embryonic leucine zipper kinase (MELK), a member of the AMP-activated protein kinase (AMPK)related kinase family, has been proposed to be involved in the control of a variety of biological processes, including the cell cycle, cell proliferation, apoptosis, signal transduction pathways, tumorigenesis, and metabolism ${ }^{1,2}$. An understanding of its multi-site protein phosphorylation, a common mechanism for regulating protein function and

\footnotetext{
Correspondence: Hyunjung Ha (hyunha@cbnu.ac.kr)

'Department of Biochemistry, School of Biological Sciences, Chungbuk National University, Cheongju 28644, Republic of Korea

Edited by A. Stephanou
}

stability, should provide insight into the mechanisms by which MPK38 regulates the activity of its substrates ${ }^{3}$. For example, MPK38 has been shown to phosphorylate Bcl-G, a pro-apoptotic protein, leading to the inhibition of BclG-induced apoptosis ${ }^{4}$. MPK38 phosphorylates ZPR9, a zinc finger protein, and stimulates its nuclear localization, which results in greater B-myb transactivation ${ }^{5}$. Conversely, MPK38 phosphorylates PDK1 at Thr354, thereby inhibiting its activity and function ${ }^{6}$. Furthermore, MPK38 phosphorylates ASK1 (Thr838), p53 (Ser15), and Smads (Ser245 of Smad2, Ser204 of Smad3, Ser343 of Smad4, and Thr96 of Smad7), activating the downstream pathways $^{2,7,8}$. Phosphorylation of serine-threonine kinase receptor-associated protein (STRAP) at Ser188 by MPK38 
activates ASK1/TGF- $\beta / \mathrm{p} 53$ signaling and inactivates PI3K/PDK1 signaling, leading to apoptotic cell death ${ }^{9}$. However, Thr76 phosphorylation of thioredoxin (Trx) by MPK38 inhibits activation of ASK1/TGF- $\beta$ / p53 signaling ${ }^{10}$. Further study of such mechanisms should greatly enhance our understanding of the biological roles of MPK38.

p21, a cyclin-dependent kinase (CDK) inhibitor, was initially isolated as a p53-inducible protein and is known to regulate the cell cycle and DNA replication. It has also been implicated in various other biological processes, including apoptosis, cell differentiation, development, tumorigenesis, and metabolism ${ }^{11,12}$. Consistent with its multiple cellular functions, a number of proteins have been found to interact with $\mathrm{p} 21$ in cells. In addition to the binding of p21 to cyclin-CDKs and proliferating cell nuclear antigen (PCNA $)^{13-15}$, which leads to the inhibition of cell cycle progression and DNA synthesis, a variety of other proteins have been found to bind to $\mathrm{p} 21$; for example, transcription factors ${ }^{16-19}$, pro-apoptotic proteins $^{20}$, protein kinases ${ }^{21-23}$, and many others ${ }^{24-28}$. p21 has also been shown to function as a positive regulator of the cell cycle; for example, p21 overexpression improves cell survival in the face of prostaglandin A2 or p53 overproduction $^{29,30}$. p21 expression is also high in various tumors, particularly in late-stage, aggressive tumors, such as glioblastoma multiforme $(\mathrm{GBM})^{31-33}$. In addition, $\mathrm{p} 21$ has been shown to play an important role in adipocyte differentiation: p21 knockdown by RNA interference in 3T3-L1 cells or its ablation in p21 $1^{-1-}$ mouse embryonic fibroblasts (MEFs) inhibits adipocyte differentiation, suggesting that $\mathrm{p} 21$ is a pro-adipogenic factor ${ }^{34}$. By contrast, other studies have shown that mice lacking p21 become obese as a result of adipocyte hyperplasia ${ }^{35}$, which implies that p21 may function as an anti-adipogenic factor. Thus, the precise role of $\mathrm{p} 21$ in adipogenesis remains controversial.

In this study, we show that MPK38 phosphorylates p21 at Thr55 and functions as a positive regulator of multiple p21 effects by stabilizing the protein. Moreover, we show that phosphorylated p21 significantly inhibits adipocyte differentiation and ameliorates obesity-induced metabolic abnormalities, implying that MPK38-induced Thr55 phosphorylation of $\mathrm{p} 21$ plays a role in the regulation of metabolism in obesity.

\section{Results}

\section{In vitro and in vivo association of p21 with MPK38}

Given that MPK38 stimulates p53 signaling ${ }^{7}$ and regulates the cell cycle ${ }^{36}$, we reasoned that MPK38 might regulate the activity of p21, a p53 target. To investigate this possibility, we first determined whether p21 physically associates with MPK38 using co-transfection experiments. p21 was only detected in the presence of glutathione S-transferase (GST)-tagged MPK38 but not control GST alone (Fig. 1a, left). An endogenous interaction of p21 with MPK38 was also detected in HEK293, NIH 3T3, and 3T3-L1 cells using co-immunoprecipitation experiments (Fig. 1a, right). We then identified the domains involved in the binding of MPK38 to p21. WT MPK38 and MPKC, which contains the carboxy-terminal domain (amino acids 270-643), bound to p21, but MCAT, which contains the $\mathrm{N}$-terminal kinase domain (amino acids 7-269), did not (Fig. 1b, left). By contrast, MPK38 interacted with the p21 deletion construct 46-71 (amino acids 46-71), which contains the CDK-binding site, but not with the 71-164 construct (amino acids 71-164) or the 141-164 construct (amino acids 141-164), which contains the PCNA binding site, in HEK293 cells (Fig. 1b, right). These results imply that the interaction is mediated via the CDK-binding region of $\mathrm{p} 21$ and the Cterminal domain of MPK38 in cells.

Next, to examine whether the interaction was direct, we performed non-denaturing polyacrylamide gel electrophoresis (PAGE) using GST-tagged recombinant MPK38 (WT, MPKC, or MCAT) and in vitro-translated ${ }^{35} \mathrm{~S}$ labeled p21. A shift in the mobility of radiolabeled p21 was only detected in the presence of recombinant WT MPK38 or MPKC but not MCAT or control GST alone, revealing that $\mathrm{p} 21$ and MPK38 directly interact (Fig. 1c). In addition, ASK1/TGF- $\beta / \mathrm{p} 53$ signals increased the formation of p21-MPK38 endogenous complexes (Fig. 1d), suggesting a critical role for stress signals in the regulation of the p21-MPK38 interaction.

\section{MPK38 phosphorylates p21 at Thr55 and stimulates its nuclear translocation}

To determine whether $\mathrm{p} 21$ can be a direct substrate for MPK38, recombinant MPK38 protein was incubated with $\left[\gamma^{-}{ }^{32} \mathrm{P}\right]$ ATP and the phosphorylation of recombinant $\mathrm{p} 21$ protein was measured. $\mathrm{p} 21$ phosphorylation was identified in the presence of MPK38 (Fig. 2a, 3rd lane). To define the MPK38 phosphorylation sites on p21, we searched for consensus MPK38 phosphorylation sites ${ }^{37}$ in p21 and performed in vitro kinase assays using three selected substitution mutants (S116A, S153A, and T55A) of p21. The T55A mutation, but not the S116A and S153A mutations, completely abolished MPK38-mediated phosphorylation (Fig. 2a, 6th lane), indicating that Thr55 of p21 is a potential MPK38 phosphorylation site. In vivo phosphorylation of $\mathrm{p} 21$ by MPK38 was confirmed using p21 knock-in (T55A) 3T3-L1 cells generated using the CRISPR/Cas9 system (Fig. 2b).

We then analyzed the effect of MPK38 on the subcellular localization of p21 because its localization is affected by phosphorylation events ${ }^{38}$. Although p21 was mainly expressed in the cytoplasm in 3T3-L1 cells, it translocated to the nucleus in the presence of WT 


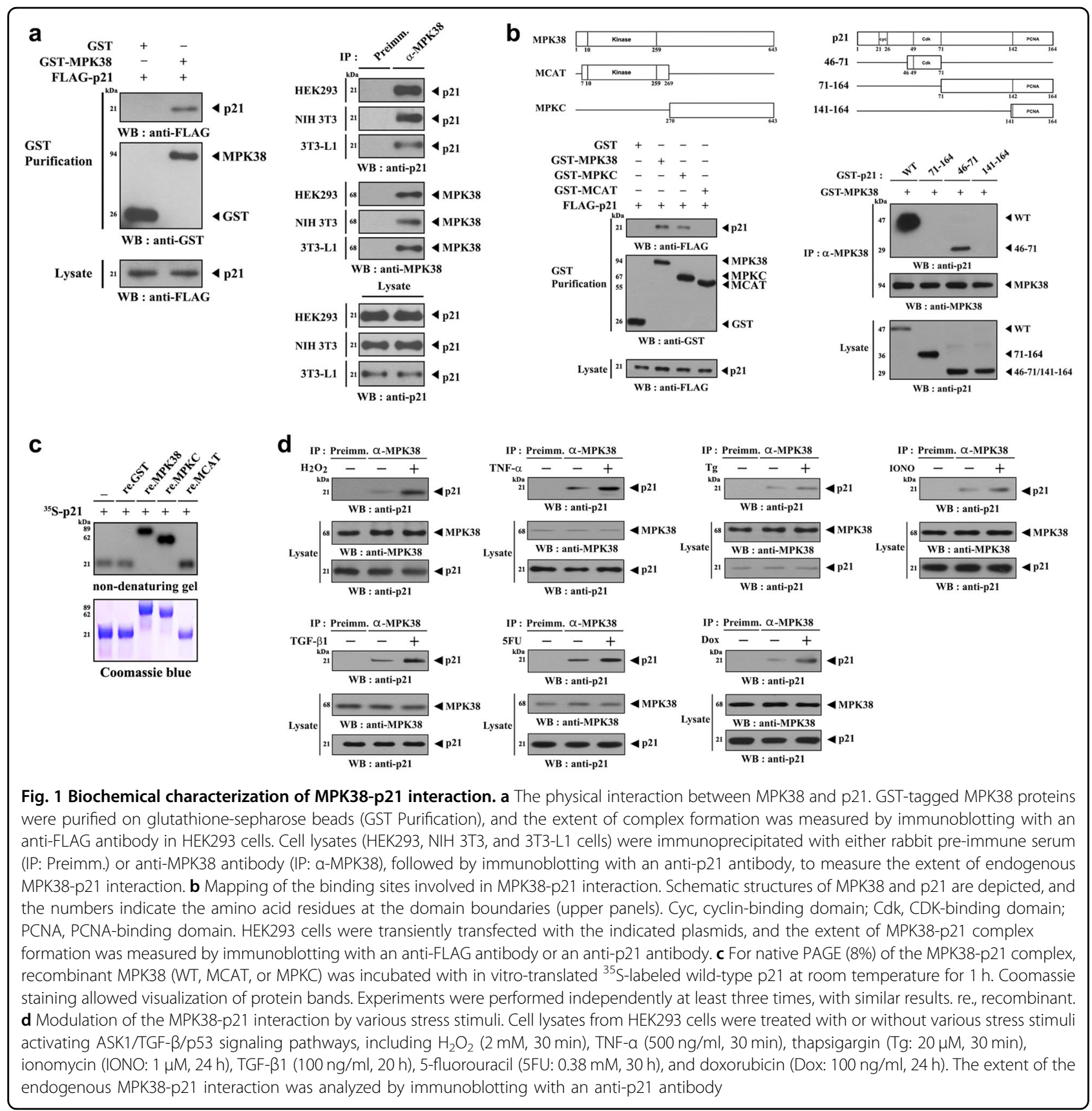

MPK38, but not kinase-dead (K40R) MPK38 (Fig. 2c, upper). This implies that Thr55 phosphorylation of $\mathrm{p} 21$ is an important trigger for the nuclear translocation of p21. However, we did not observe any change in the intracellular distribution of the T55A mutant under the same conditions (Fig. 2c, lower). MPK38-induced nuclear localization of $\mathrm{p} 21$ was also demonstrated using immunofluorescence microscopy (Fig. 2d). These results suggest that Thr55 phosphorylation of p21 triggers the translocation of $\mathrm{p} 21$ from the cytoplasm to the nucleus.

\section{MPK38 reduces the assembly of both the CDK2-cyclin E and CDK4-cyclin D complexes}

p21 has been shown to inhibit the assembly and activation of both the CDK2-cyclin E and CDK4-cyclin D complexes $^{15}$. To determine whether MPK38 affects the formation of these complexes, we analyzed their assembly in the presence of MPK38. MPK38 reduced the assembly of both complexes in a dose-dependent manner, whereas K40R MPK38 had no such effect (Fig. 3a). Also, MPK38 increased the binding of p21 to CDK2 or CDK4 in a kinase-dependent manner (Fig. 3b), suggesting that 


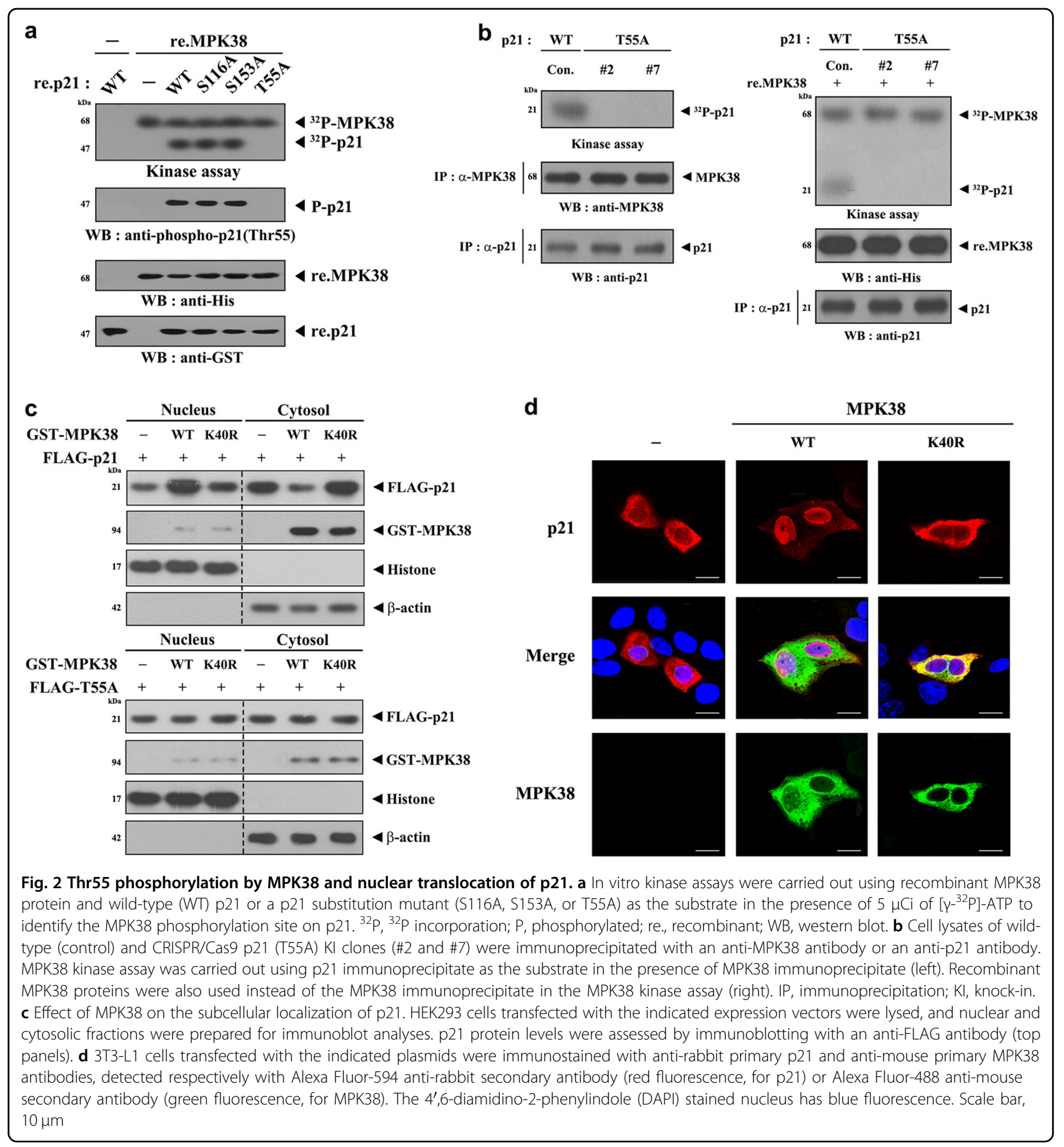

phosphorylated p21 is capable of interacting with and inhibiting CDK2 or CDK4, and possibly inhibiting cell cycle progression. To confirm this, the association of $\mathrm{p} 21$ with CDK2 or CDK4 was analyzed using both WT and T55A p21, and indeed these complexes were more common in the presence of WT p21 than in the presence of the T55A mutant (Fig. 3c). These data suggest that MPK38 interacts with and phosphorylates p21, resulting in the induction of CDK2-p21 and CDK4-p21 complex formation, and subsequent inhibition of the assembly of CDK2-cyclin E and CDK4-cyclin D complexes.

MPK38 stimulates $\mathrm{p} 21$ promoter activity, and p21mediated apoptosis and growth arrest, in a kinasedependent manner

To explore the functional significance of the p21MPK38 interaction, we first performed reporter assays to quantify the effect of MPK38 on p53/p300-induced p21 


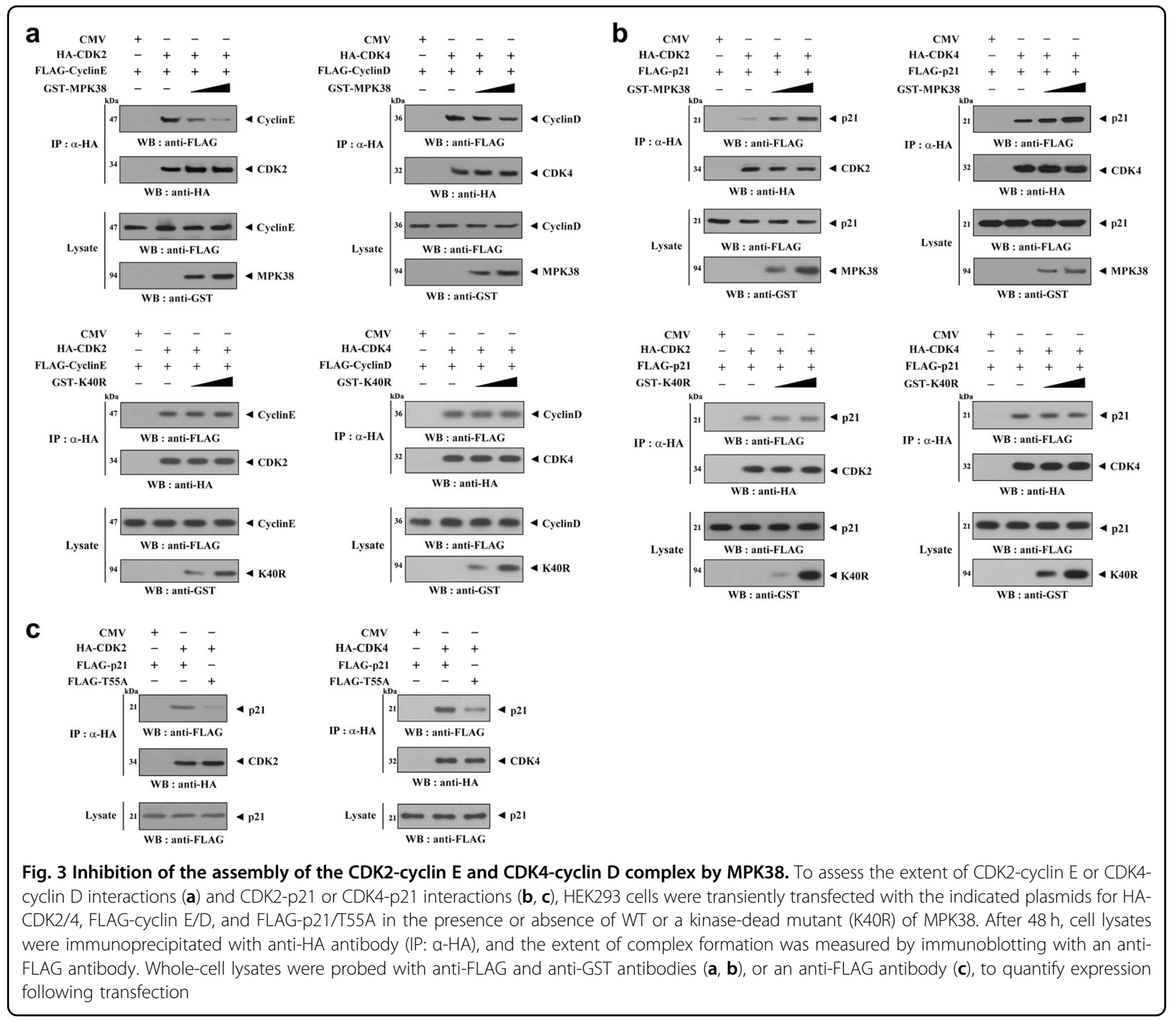

promoter activity because $\mathrm{p} 21$ increased the formation of complexes of p53 and its coactivator, p300, in an MPK38 phosphorylation-dependent manner (Fig. S1). We found that MPK38 increased p21 promoter activity in a kinaseand dose-dependent manner (Fig. 4a). This suggests that the kinase activity of MPK38 is important for the regulation of $\mathrm{p} 21$ function. To confirm this positive influence of MPK38 on p21 promoter activity, we carried out a knockdown experiment using MPK38-specific siRNAs and found that down-regulation of endogenous MPK38 reduced p21 promoter activity in a dose-dependent manner (Fig. 4b). In a separate experiment using two MPK38 deletion constructs (MCAT and MPKC), MPKC dose-dependently stimulated p21 promoter activity (Fig. 4c). However, this effect was not observed in the presence of MCAT, which fails to bind to p21 (Fig. 1b). This indicates that both direct interaction and phosphorylation of p 21 by MPK38 play important roles in the upregulation of p21 promoter activity. Consistent with this, MPK38 increased p21-mediated apoptosis in a kinase-dependent manner, but this stimulatory effect did not occur in the presence of the T55A mutation (Fig. 4d).

Next, to determine whether MPK38 influences p21induced cell cycle arrest, we performed flow cytometry analysis using H1299 cells. As shown in Fig. 4e, a much larger number of cells ( $46 \%$ vs. $~ 30 \%$ ) was found to be in G1 phase after co-transfection with MPK38 and p21 than in controls transfected with p21 alone. However, this effect was barely detectable in the presence of the T55A mutant ( 27\% vs. $\sim 25 \%)$. These findings suggest that MPK38 stimulates p21-mediated phenomena, such as apoptosis and cell cycle arrest, in a kinase-dependent manner. 

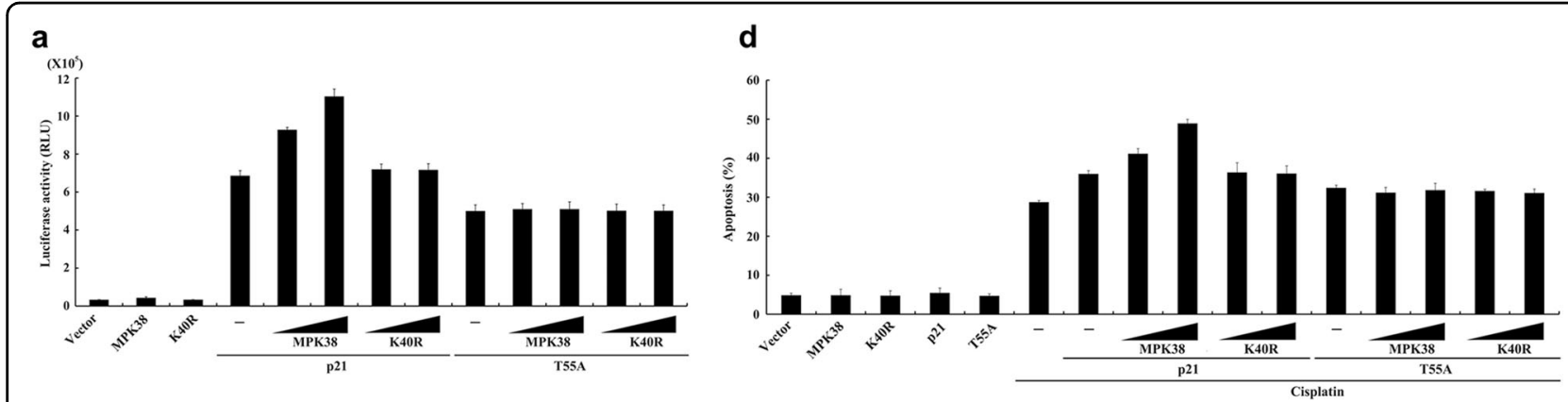

b

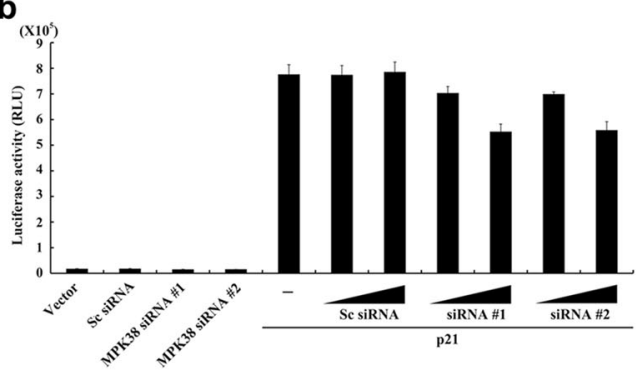

e

\begin{tabular}{c|ccccccc}
\hline Cell cycle & Vector & $\mathrm{p} 21$ & $\mathrm{p} 21$ MPK38 (1) & $\mathrm{p} 21$ MPK38 (2) & T55A & T55AMPK38 (1) & T55AMPK38 (2) \\
\hline G1 (\%) & 23.2 & 30.3 & 36.2 & 45.7 & 25.3 & 26.3 & 27.4 \\
S (\%) & 50.2 & 40.3 & 35.2 & 24.3 & 48.5 & 44.7 & 43.8 \\
G2 $\%$ (\%) & 26.6 & 29.4 & 28.6 & 30 & 26.2 & 29 & 28.8 \\
\hline
\end{tabular}

C
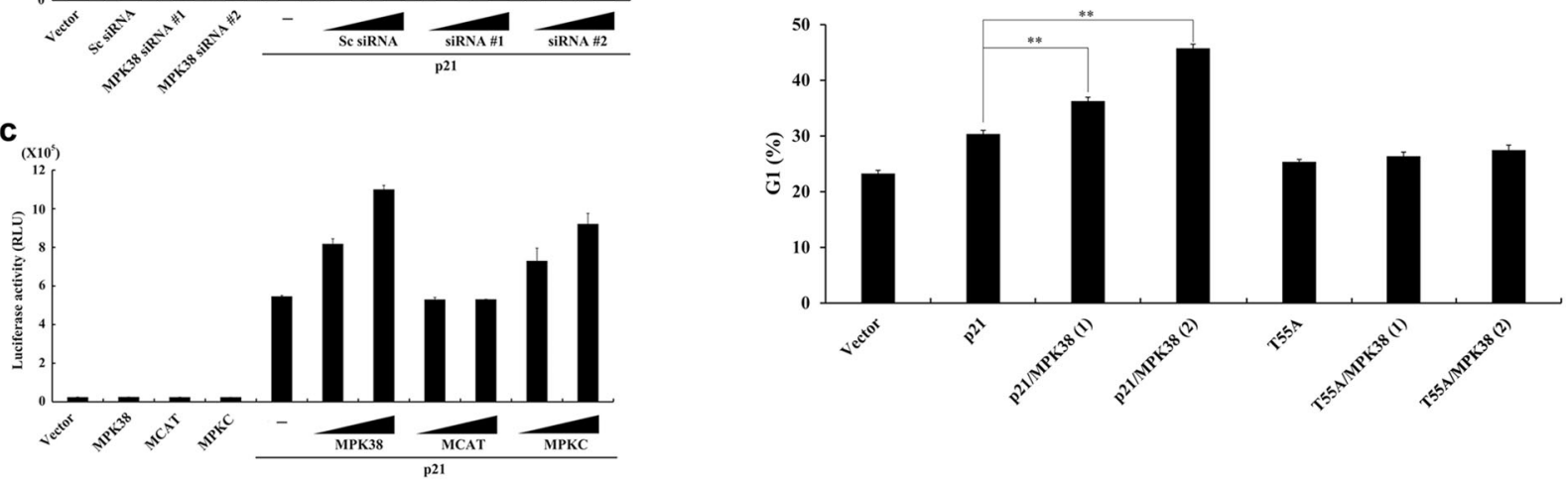

Fig. 4 Stimulation of p21 promoter activity, and p21-mediated apoptosis and growth arrest, by MPK38. a-c Stimulation of p21 promoter activity by MPK38. HCT116 cells expressing wild-type p53, required for p53/p300-induced p21 promoter activity, were transiently transfected with WWP-Luc plasmid $(0.2 \mu \mathrm{g})$ and two different amounts of MPK38 (WT and K40R: 0.4 or $0.8 \mu \mathrm{g})$, MCAT and MPKC $(0.4$ or $0.8 \mu \mathrm{g})$, MPK38-specific siRNAs (\#1 or \#2: 100 or $200 \mathrm{nM})^{6}$, and scrambled siRNA (Sc: 100 or $\left.200 \mathrm{nM}\right)^{6}$, as indicated. The data shown are means ( \pm SEM) of three independent experiments. d Stimulation of p21-mediated apoptosis by MPK38. MCF7 cells expressing wild-type p53, an important mediator of cisplatin-induced apoptosis, were transiently transfected with p21 (WT and T55A: $0.4 \mu \mathrm{g}$ ) and MPK38 (WT and K40R: $0.3 \mathrm{or} 0.6 \mu \mathrm{g}$ ) in the presence or absence of cisplatin $(1 \mathrm{\mu g} / \mathrm{ml})$. GFP expression system was used to detect cell death ${ }^{2}$. e Stimulation of p21-mediated growth arrest by MPK38. p53-null H1299 cells were transfected with p21 (WT and T55A: $0.4 \mu \mathrm{g}$ ) and MPK38 (WT: 0.3 or $0.6 \mu \mathrm{g}$ ), and fixed for FACS analysis $40 \mathrm{~h}$ after transfection and $16 \mathrm{~h}$ after nocodazole treatment. The data are also shown in the graph where $y$-axis indicates the percentage of cells in G1 phase. ${ }^{* *} p<0.01$ compared to control expressing p21. MPK38 (1) and (2) indicate the use of $0.3 \mu \mathrm{g}$ and $0.6 \mu \mathrm{g}$ of MPK38, respectively

\section{MPK38 potentiates p21-mediated inhibition of adipocyte differentiation}

p21 is known to be a critical regulator of adipocyte differentiation, which involves a sequence of cell cycle $\operatorname{arrests}^{34,35}$. To determine the effects of p21 on the differentiation of 3T3-L1 adipocytes, WT and T55A p21 were overexpressed before the induction of their differentiation. The expression of WT p21 suppressed adipocyte differentiation, as assessed using Oil Red $\mathrm{O}$ staining. However, no such effect was observed in the presence of the T55A mutant (Fig. 5a, top and middle). The expression of WT p21 was accompanied by lower expression of PPAR $\gamma$ and CCAAT-enhancer-binding protein $\alpha(\mathrm{C} /$ $\mathrm{EBP} \alpha)$, which are involved in adipocyte differentiation, along with lower expression of the mature adipocyte marker perilipin (Fig. 5a, bottom). In addition, the mRNA expression of other key adipogenic genes, such as fatty acid binding protein 4 (Fabp4) and adiponectin (Adipoq), was also lower in 3T3-L1 cells expressing WT p21 than in control cells expressing an empty vector alone (Fig. S2). However, these differences in expression were barely detectable in cells expressing T55A p21. The importance of MPK38-mediated Thr55 phosphorylation of p21 in the inhibition of adipocyte differentiation was also verified using 3T3-L1 cells transfected with p21 alone, or cotransfected with p21 and MPK38, as assessed using Oil Red $\mathrm{O}$ staining and immunoblot analysis (Fig. 5b). This finding was further confirmed using MEFs from p21-null 
a
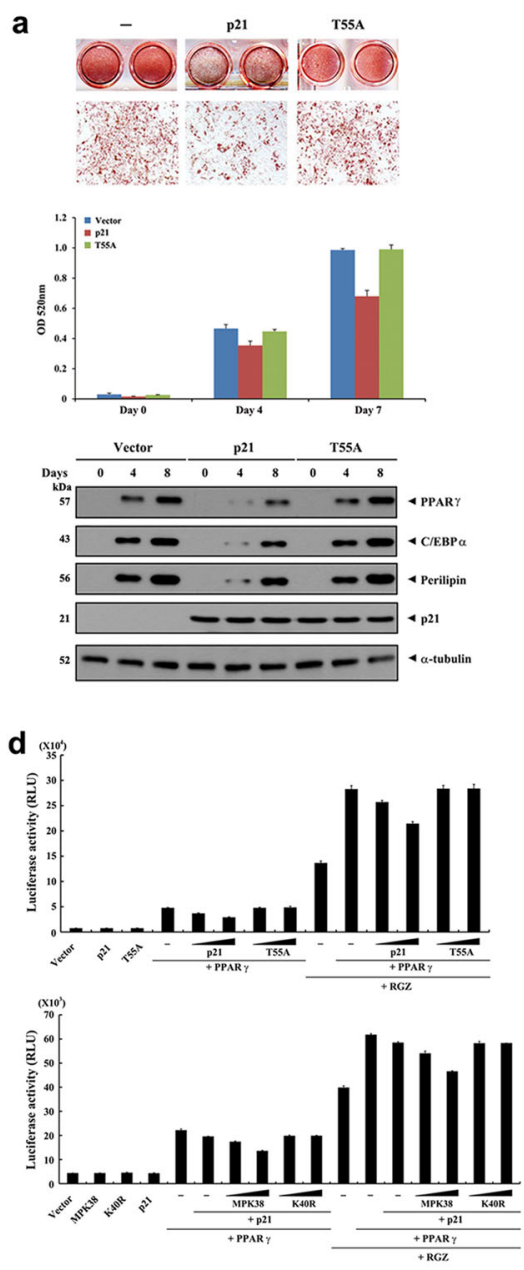

b
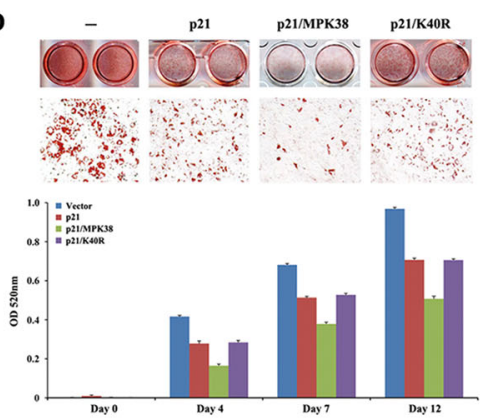

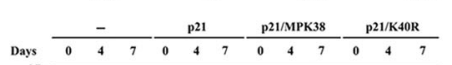
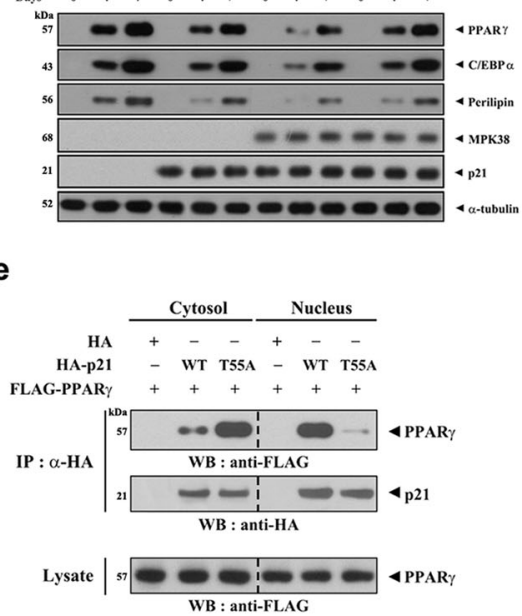
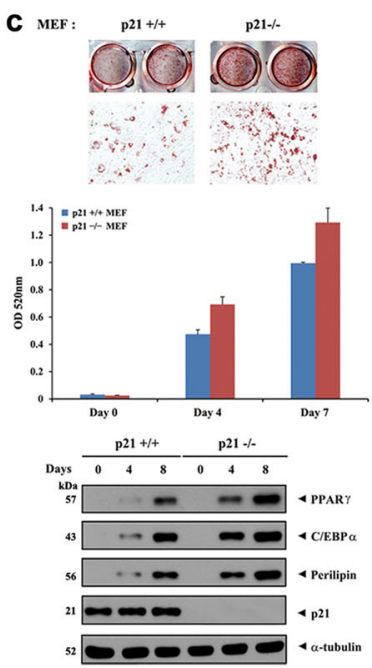

f

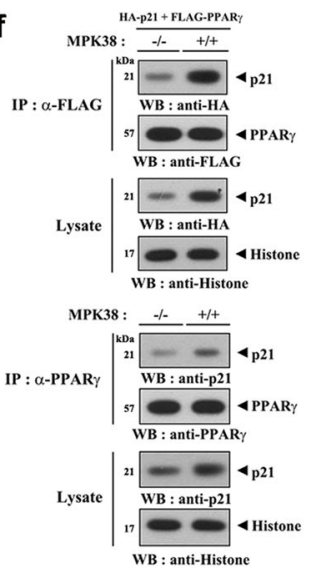

Fig. 5 Inhibition of adipocyte differentiation by Thr55 phosphorylation of p21. a-c Effect of Thr55 phosphorylation of p21 on adipocyte differentiation. Oil Red O staining (top), measurements of absorbance at $520 \mathrm{~nm}$ (middle), and immunoblot analyses (bottom) were performed using 3T3-L1 preadipocytes transfected with the indicated plasmids $(\mathbf{a}, \mathbf{b})$ or MEF cells expressing p21 (+/+) or not $(-/-)$ (c) on the indicated number of days after the start of differentiation. Oil Red $\mathrm{O}$ staining of cells 10-14 days after the start of differentiation is shown. $\mathbf{d}$ Effect of Thr55 phosphorylation of p21 on PPARY-mediated transactivation. 3T3-L1 cells were transfected with $0.2 \mu \mathrm{g}$ PPARY luciferase plasmid, two different amounts of p21 (WT and T55A: 0.1 or $0.2 \mu \mathrm{g}$ ) and MPK38 (WT and K40R: 0.1 or $0.2 \mu \mathrm{g})$, p21 $(0.2 \mu \mathrm{g})$, or PPARy $(0.3 \mu \mathrm{g})$ in the presence or absence of RGZ $(10 \mu \mathrm{M})$. The data shown are means $( \pm$ SEM) of three independent experiments. e Effect of Thr55 phosphorylation on the extent of p21-PPARY complex formation in the nucleus. HEK293 cells transiently transfected with the indicated expression vectors were lysed, and nuclear and cytosolic fractions were prepared for immunoblot analysis. The extent of p21-PPARy complex formation was assessed by immunoblotting with an anti-FLAG antibody (top panel). $\mathbf{f}$ Quantification of exogenous (upper) and endogenous (lower) p21-PPARy complex formation using MEF cells expressing MPK38 $(+/+)$ or not $(-/-)$

mice because $\mathrm{p} 21^{-1-}$ MEFs displayed a greater degree of adipocyte differentiation than control $\mathrm{p} 21^{+/+}$MEFs (Fig. 5c).

To elucidate how p21 inhibits adipogenesis, we assessed the transcriptional activity of PPAR $\gamma$, a key transcription factor in adipogenesis ${ }^{39-41}$, using a luciferase assay with p21 in the presence of rosiglitazone (RGZ), a PPARy agonist. We found that WT p21 suppressed the transcriptional activity of PPAR $\gamma$ in a dose-dependent manner, whereas the T55A mutant, which is unable to translocate to the nucleus (see Fig. 2c), did not have a suppressive effect under the same conditions (Fig. 5d, upper). The importance of Thr55 phosphorylation of $\mathrm{p} 21$ in the suppression of PPAR $\gamma$ transcriptional activity was also demonstrated using a reporter assay with MPK38 in the presence of p21 (Fig. 5d, lower). These findings indicate that nuclear translocation of $\mathrm{p} 21$ is important for the negative regulation of PPARy. Indeed, the WT p21 strongly bound to PPAR $\gamma$ in the nucleus, but not in the cytoplasm (Fig. 5e). Consistent with this, MPK38 $8^{+/+}$ 
MEFs displayed greater exogenous and endogenous complex formation between p21 and PPAR $\gamma$ in the nucleus than MPK38 $8^{-1-}$ MEFs (Fig. 5f), suggesting that p21 may function as a repressor of PPAR $\gamma$. These results imply that MPK38 negatively regulates adipogenesis by promoting the association of $\mathrm{p} 21$ with PPAR $\gamma$ in the nucleus, thereby preventing the transcriptional activity of PPARY.

\section{Thr55 phosphorylation of $\mathrm{p} 21$ contributes to the amelioration of adiposity and impaired glucose and energy metabolism in diet-induced obese mice}

Our previous studies have shown that obesity is inversely associated with MPK38 and p21 expression in mice ${ }^{42}$. Therefore, an adenoviral delivery system was employed to investigate whether the restoration of p21 expression would ameliorate the obesity-induced metabolic abnormalities in male C57BL/6 mice fed an HFD. The adenoviral delivery of p21 (Ad-p21) reduced the number of large hypertrophic adipocytes (Fig. 6a) and the mRNA expression of key adipogenic regulators (Fig. 6b), including $\mathrm{C} / \mathrm{EBP} \alpha, \mathrm{PPAR} \gamma$, and FABP4, when compared with uninfected or Ad-GFP-infected HFD-fed C57BL/6 mice. However, Ad-T55A infection had no such effect, implying a significant role of Thr 55 phosphorylation of p21 in the regulation of adipocyte metabolism and obesity.

Ad-p21 infection improved glucose tolerance and insulin sensitivity in HFD-fed C57BL/6 mice (Fig. 6c, d). In parallel, Ad-p21 infection caused lower circulating glucose and insulin concentrations under fasting conditions (Fig. 6e, f) and stimulated in vitro insulin-stimulated 2-deoxy-glucose uptake into white adipose tissue (WAT) and muscle (Fig. 6g, left). Ad-p21 infection also increased activation of the insulin receptor substrate (IRS)-phosphoinositide 3-kinase (PI3K) pathway, which stimulates glucose uptake (Fig. 6g, right). Ad-p21 infection caused considerably lower levels of blood glucose (Fig. 6h) and mRNA expression of gluconeogenic genes in liver (Fig. 6i). However, none of these effects were observed after Ad-T55A infection. Such beneficial effects of the adenoviral delivery of p21 were also observed in p21-deficient obese mice fed an HFD (Fig. S3). Regarding energy metabolism, the HFD-fed mice infected with Ad-p21 had increased $\mathrm{O}_{2}$ consumption and $\mathrm{CO}_{2}$ production (Fig. S4a, b) and exhibited a higher rate of energy expenditure, which was associated with a change in locomotor activity (Fig. S4d, g). Ad-p21 infection altered energy substrate usage, as evidenced by a higher level of respiratory exchange ratio (RER) (Fig. S4c). However, Ad-p21 infection did not affect food and water intake (Fig. S4e, f). All these results indicate that p21 ameliorates defects in adipocyte and glucose and energy metabolism in a MPK38 phosphorylation-dependent manner in dietinduced obese mice.

\section{Thr55 phosphorylation of p21 contributes to the amelioration of abnormal lipid metabolism and inflammation in diet-induced obese mice}

We next determined whether p21 regulates the expression of lipogenic genes in HFD-fed C57BL/6 mice. Consistent with the lower circulating free fatty acid (FFA) concentration, Ad-p21 infection reduced the mRNA expression of lipogenic genes in liver and WAT (Fig. 7a). Ad-p21 infection reduced lipogenesis and liver triglyceride content, as well as circulating total cholesterol, highdensity lipoprotein (HDL)-cholesterol, and low-density lipoprotein (LDL)-cholesterol concentrations (Fig. 7a, b). We also quantified the expression of proinflammatory genes in serum because cholesterol and fatty acids are involved in inflammatory pathways ${ }^{43}$. Ad-p21 infection considerably reduced the serum concentrations of proinflammatory proteins (Fig. 7c) and the mRNA expression of proinflammatory genes in WAT (Fig. S5), but Ad-T55A infection did not have these effects.

Ad-p21 infection increased the mRNA expression of lipolytic genes in WAT and the hepatic fatty acid utilization by enhancing the mitochondrial and peroxisomal pathways of fatty acid oxidation (Fig. 7d, e). Ad-p21 infection resulted in higher expression of key genes involved in fatty acid oxidation in WAT as well as lower serum triglyceride concentration and greater isoproterenol-stimulated lipolytic activity (Fig. 7f). Consistent with lower liver triglyceride, Ad-p21 infection decreased substantially liver lipid accumulation (Fig. 7a, g). However, none of these effects were observed after AdT55A infection. All these data are consistent with Thr55 phosphorylation of p21 upregulating lipid metabolism.

We next investigated whether $\mathrm{p} 21$ regulates ketogenesis in the liver. Ad-p21 infection stimulated ketone body production (Fig. 7h) and ketogenic gene expression (Fig. 7i) in response to fasting. Previous studies have implicated ketogenesis in the modulation of cell signaling pathways $^{44}$, and indeed Ad-p21 infection caused lower levels of phospho-S6 Ser240/244 in response to fasting (Fig. 7j, left). Consistent with this, Ad-p21 infection downregulated mechanistic target of rapamycin (mTORC) 1 signaling, and this demonstrated a reciprocal trend to ketogenesis (Fig. 7j, right). Similar effects were identified in p21-deficient obese mice fed an HFD (Fig. S6). These results suggest that $\mathrm{p} 21$ can ameliorate the hyperlipidemic phenotype of HFD-fed mice in a MPK38 phosphorylation-dependent manner.

\section{MPK38 increases p21 stability}

The transcriptional activation and post-translational modifications play important roles in p21 degradation, although the precise mechanism leading to protein degradation is still controversial ${ }^{45-47}$. To determine whether the high expression of p21 in MPK38-transfected 


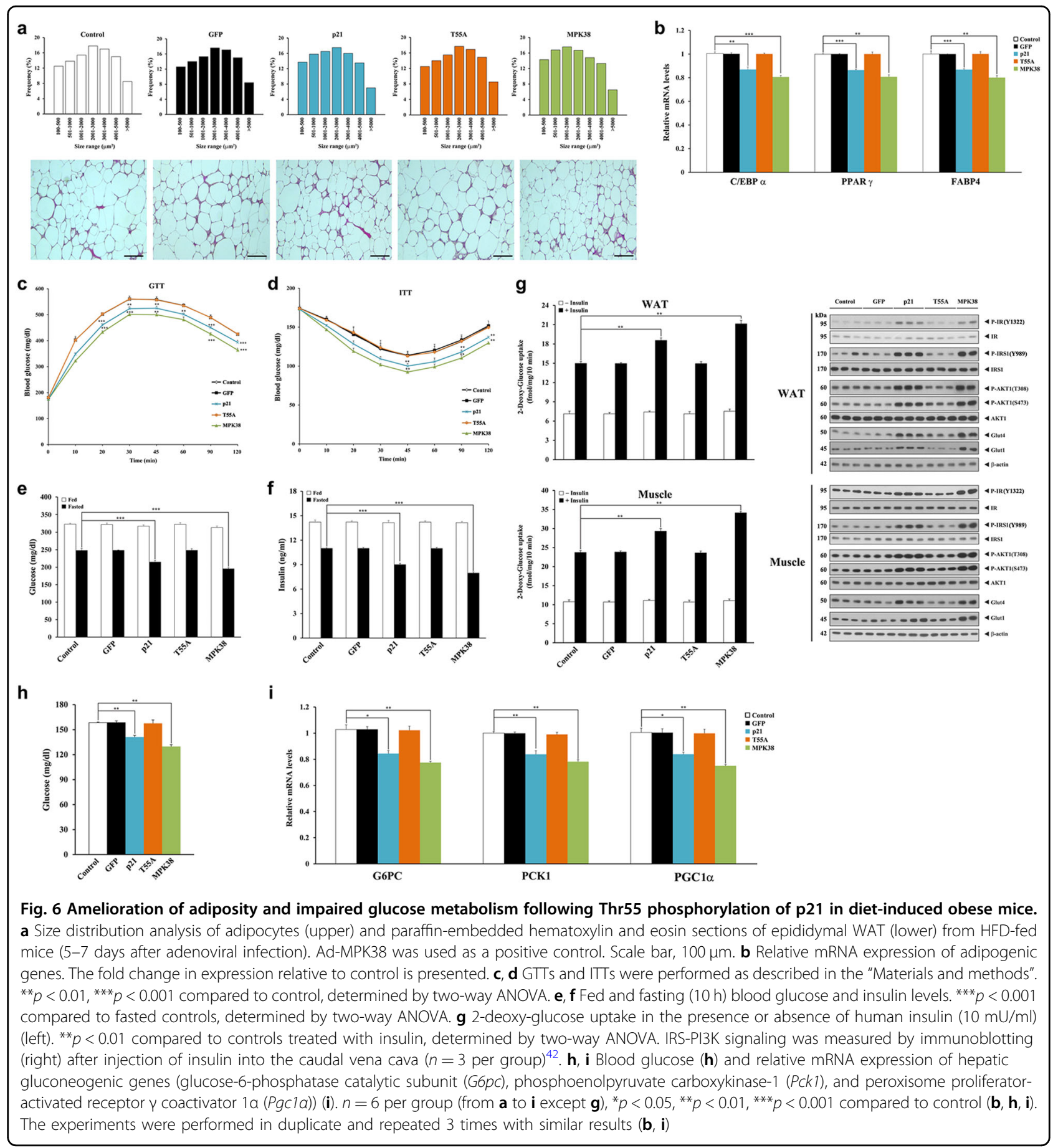

cells (Fig. 8a) is due to protein stabilization, p21 stability was first assessed by immunoblot analysis. p21 was degraded after cycloheximide treatment in either untransfected or K40R MPK38-transfected cells. By contrast, p21 was stabilized in cells transfected with WT MPK38 (Fig. 8b). Moreover, treatment with both cycloheximide and MG132, a proteasomal inhibitor, led to greater stability of p21 than in HEK293 cells that were not treated with MG132, implying an important role for the proteasome pathway in p21 degradation (Fig. 8b). We next analyzed the effect of MPK38 on p21 ubiquitination and found that MPK38 dose-dependently reduced the ubiquitination of $\mathrm{p} 21$ in a kinase-dependent manner (Fig. 8c). We also determined whether the regulation of p21 stability by MPK38 is dependent on the documented interaction between $\mathrm{p} 21$ and $\mathrm{Mdm} 2^{48}$, and found that 


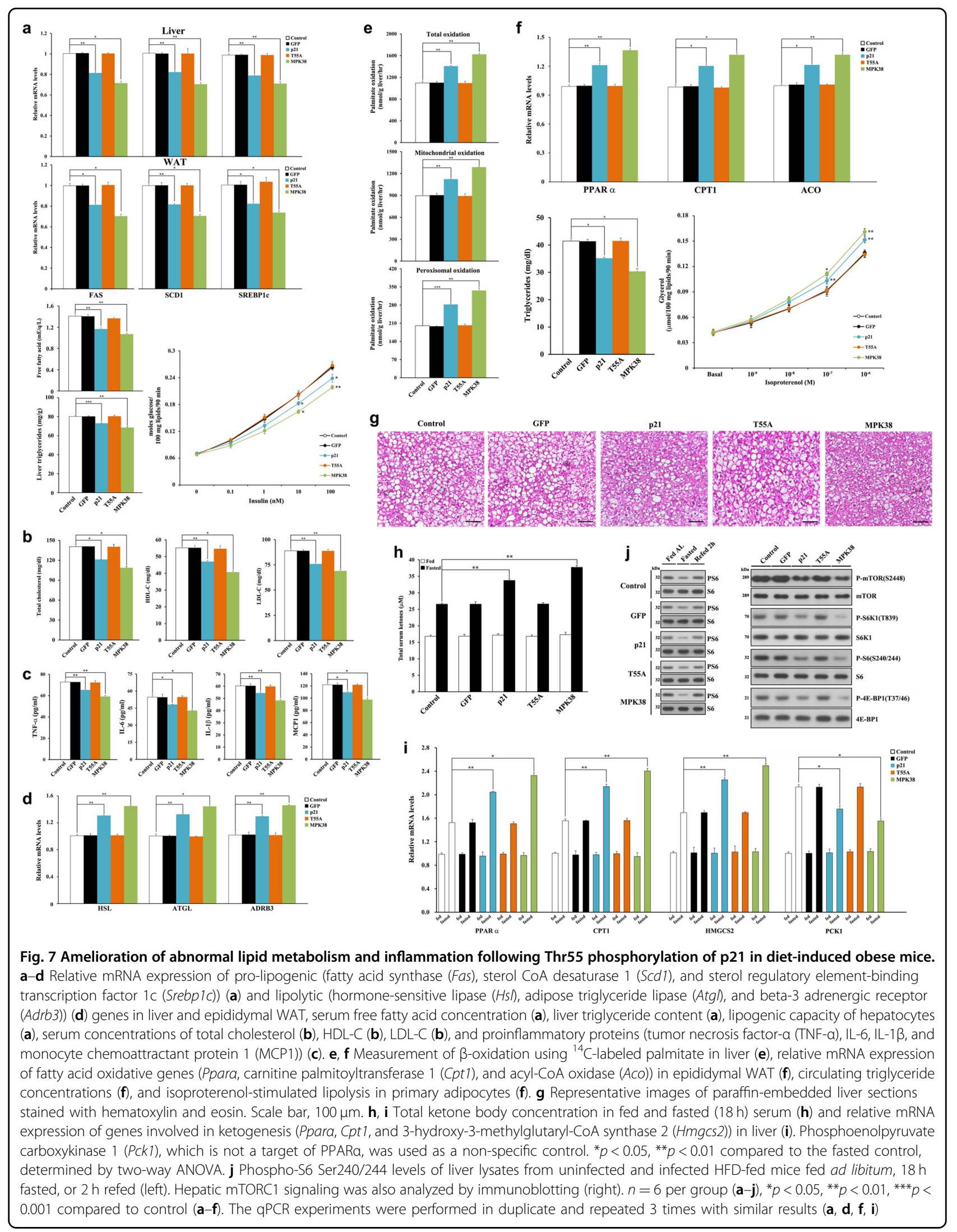


a

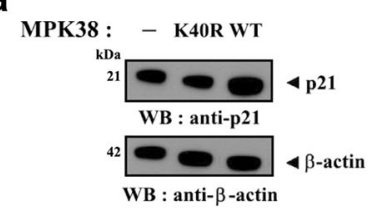

b

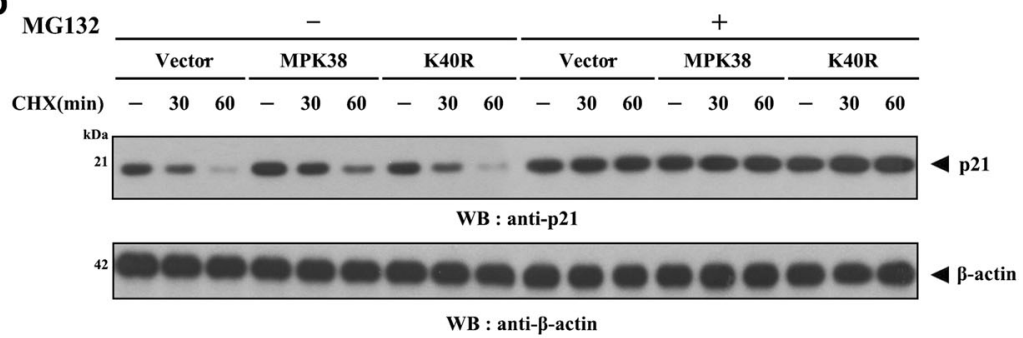

d

C
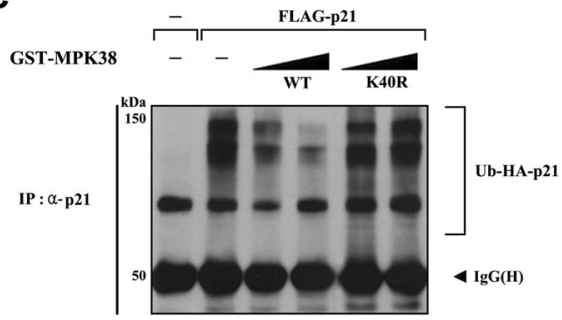

WB : anti-HA

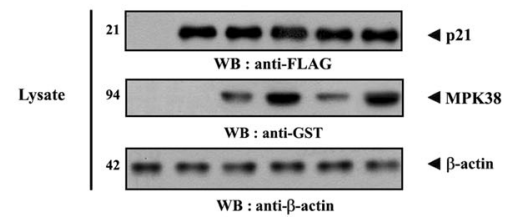

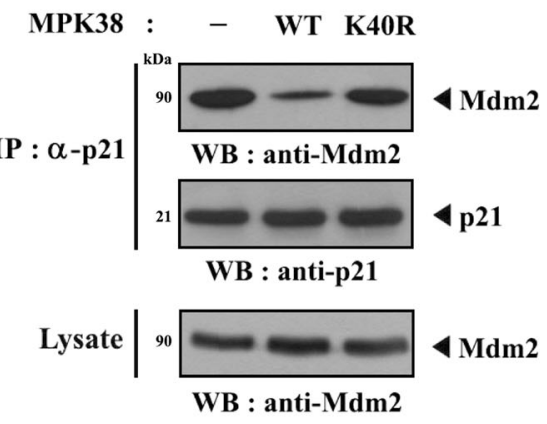

Fig. 8 Stabilization of p21 by MPK38. a, b p21 protein levels were evaluated by immunoblotting with an anti-p21 antibody. HEK293 cells transfected with vectors encoding MPK38 (WT or K40R) or empty vector (vector) were treated with or without MG132 (10 $\mu \mathrm{M})$ in the presence of cycloheximide $(\mathrm{CHX}, 20 \mathrm{\mu g} / \mathrm{ml})$ for the indicated time intervals. c The ubiquitination of endogenous p21 was assessed in HEK293 cells transfected with increasing amounts of WT and K40R MPK38, HA-tagged ubiquitin (Ub), and FLAG-tagged p21 as indicated. d HEK293 cells were transfected with vectors encoding MPK38 (WT or K40R), and the extent of endogenous p21-Mdm2 complex formation was measured by immunoblot analysis using an anti-Mdm2 antibody

MPK38 markedly reduced endogenous p21-Mdm2 complex formation in a kinase-dependent manner (Fig. 8d). These findings suggest that MPK38 promotes p21 stability through Thr55 phosphorylation.

\section{Discussion}

In this study, we explored the role of MPK38, also known as MELK, on p21 activity and function, and found that MPK38 positively regulates $\mathrm{p} 21$-mediated apoptosis, cell cycle arrest, and metabolic regulation by phosphorylating p21 at Thr55. Together with the previous finding that MPK38 activates p53 signaling through direct phosphorylation of $\mathrm{p} 53$ at Ser $15^{7}$, our current results indicate that MPK38 stimulates the activity of p21 through both p53-dependent and -independent mechanisms. In order to elucidate the regulatory mechanism of MPK38 on p21 activity, we investigated whether the physical interaction between the two and the phosphorylation of p21 by MPK38 (Figs. 1 and 2) are important for the regulation of p21 activity using diverse biochemical techniques and an adenoviral delivery system in diet-induced obese mice. Our findings imply that Thr55 phosphorylation of $\mathrm{p} 21$ by
MPK38 may play a crucial role in the regulation of p21 activity. To verify this, we performed reporter assays to assess p21 promoter activity in the presence of MPK38, and found that MPK38 stimulates $\mathrm{p} 21$ promoter activity in a kinase-dependent manner (Fig. 4a). MPK38 also stimulated p21-mediated cell cycle arrest in a kinase-dependent manner, suggesting a potential role of MPK38 in adipocyte differentiation (Fig. 4e). Consistent with this, MPK38 kinase-dependently contributed to the p21-mediated metabolic regulation in obesity (Figs. 6 and 7).

p21 is known to be an unstable protein, having a halflife of about $30 \mathrm{~min}$, and is degraded proteasomally in both an ubiquitin-dependent and an ubiquitinindependent manner ${ }^{46,47}$. Our results show that MPK38 inhibits Mdm2-dependent p21 ubiquitination via Th55 phosphorylation of p21 (Fig. 8c). Recent studies have also shown that p21 can be regulated by post-translational mechanisms $^{38}$. For example, Ser146 phosphorylation by $\mathrm{AKT} /$ protein kinase $\mathrm{B}(\mathrm{PKB})$ stabilizes p21, whereas p21 is destabilized by glycogen synthase kinase (GSK) $3 \beta$-mediated phosphorylation at $\operatorname{Thr} 57^{49,50}$. However, Thr145 phosphorylation by AKT/PKB does not affect 
p21 stability but causes its cytoplasmic translocation ${ }^{51}$. Similarly, Ser153 phosphorylation by dual specificity tyrosine-phosphorylation-regulated kinase 1B (Dyrk1B) stimulates the translocation of p21 from the nucleus to the cytoplasm ${ }^{52,53}$. The present study has shown that MPK38 is capable of inducing greater stability and nuclear translocation of p21 through Thr55 phosphorylation. By contrast, the stability and subcellular localization of p21 are not affected by CDK and c-Jun N-terminal kinase (JNK)/p38-mediated Ser130 phosphorylation ${ }^{54}$.

Adipogenesis is tightly controlled by intricate transcription factor networks operating at different time points during differentiation ${ }^{55,56}$. PPAR $\gamma$ is considered a master regulator of adipogenesis from both in vitro and in vivo studies. Indeed, PPAR $\gamma$ is required for adipocyte differentiation ${ }^{57,58}$, and in many cases its expression is sufficient for the differentiation of non-adipose cells into adipocyte-like cells ${ }^{59,60}$. PPAR $\gamma$ also regulates insulin sensitivity, lipogenesis, and adipocyte survival and func$\operatorname{tion}^{61}$. Thus, it is reasonable proposition that $\mathrm{p} 21$, a transcriptional regulator, could regulate adipocyte differentiation by affecting transactivation by PPAR $\gamma$. In the present study, we found that MPK38 plays a critical role in the association between $\mathrm{p} 21$ and PPAR $\gamma$, following Thr55 phosphorylation of $\mathrm{p} 21$. Indeed, phosphorylated p21 strongly interacted with PPAR $\gamma$ in the nucleus, leading to inhibition of PPAR $\gamma$ binding to peroxisome proliferator response elements (PPRE) in target genes (Fig. 5d-f). This finding suggests a model in which p21 inhibits adipocyte differentiation by preventing PPAR $\gamma$ transcriptional activity as a result of a direct interaction with PPARY in the nucleus (Fig. S7).

In conclusion, our findings demonstrate that MPK38 plays a key role in the positive regulation of p21 activity and function by phosphorylating p21 on Thr55, and suggest that MPK38 is a positive regulator of $\mathrm{p} 21$. However, further investigation of the effect of p21 phosphorylation at other sites directly related to its activity is necessary to clarify the molecular mechanisms of the regulation of obesity-associated metabolic changes by $\mathrm{p} 21$.

\section{Materials and methods}

Antibodies, plasmids, chemicals, MEF cells, oligonucleotides, and biochemical analyses

The antibodies, plasmids, and chemicals used have been described previously ${ }^{8,42,62,63}$. The anti-phospho-p21(T55) antibody was raised against the synthetic phosphopeptide FDFVTETPL, in which $\underline{T}$ represents phosphothreonine (Young In Frontier, Seoul, Korea), in a rabbit. The WWPLuciferase ( $\mathrm{p} 21 / \mathrm{WAF} 1$ promoter) plasmid containing the p53-binding site was from Addgene (no. 16451). $\mathrm{MEF}^{\mathrm{p} 21-1-}$ cells were generated after timed matings of homozygous p21 and MEF ${ }^{\text {MPK38-I- }}$ has been described previously ${ }^{42}$. The oligonucleotides were from Bioneer Ltd (Cheongwon, Korea). Biochemical analyses, including coimmunoprecipitation, immunoblot analysis, luciferase assay, and in vitro kinase assay for MPK38, as well as quantitative real-time PCR (qPCR), confocal microscopy, and assays for apoptosis and cell cycle arrest, were performed using the indicated cells and experimental conditions, as previously described ${ }^{2,8,42}$.

\section{Construction of MPK38-mediated phosphorylation- defective p21 mutants}

To achieve three p21 mutants (substitution of alanine for serine or threonine residues), wild-type p21 was used as the template for PCR with either the p21 forward or reverse primer (forward, 5'-GCGAATTCATGTCAGAAC CGGCTGGG-3'; reverse, 5'-GCCTCGAGTTAGGGCTT CCTCTTGGA-3'; EcoRI/XhoI site underlined), together with one of the following pairs of primer sequences: for S116A, sense 5'-GTGGACCTGTCACTGGCTTGTACC CTTGTGCCT-3', antisense 5'-AGGCACAAGGGTAC AAGCCAGTGACAGGTCCAC-3'; for S153A, sense 5'ACAGATTTCTACCACGCCAAACGCCGGCTGATC-

3', antisense 5'-GATCAGCCGGCGTTTGGCGTGGTAG AAATCTGT-3'; and for T55A, sense 5'-AACTTCGAC TTTGTCGCCGAGACACCACTGGAG-3', antisense 5' CTCCAGTGGTGTCTCGGCGACAAAGTCGAAGTT-

3'. The PCR products were cut with EcoRI and XhoI and then subcloned into pGEX4T-1 vector using EcoRI/XhoI site to generate pGEX4T-1-p21 substitution mutants.

\section{Generation of a p21 knock-in (T55A) cell line}

Genome editing with the CRISPR/Cas9 system was performed in 3T3-L1 cells, as previously described ${ }^{62,63}$. Single-guide (sg) RNA (5'-AATGGCGGGCTGCATCCAGG-3') was designed to target the genome adjacent to the p21 mutation site. The annealed oligonucleotides (5'CACCGAATGGCGGGCTGCATCCAGG-3' and 5'AAACCCTGGATGCAGCCCGCCATTC-3') containing the p21 guide sequence and Bbs1 ligation adapters were ligated into pX458 vector (Addgene) using $5 \mu$ of $2 \times$ Quick-Ligation Buffer and $1 \mu \mathrm{l}$ of QuickLigase (New England BioLabs, Ipswich, MA). 3T3-L1 cells were cotransfected with $1 \mu \mathrm{g}$ p21 sg RNA plasmid and pUC19 p21 T55A to generate the p21 knock-in (T55A) cell line. The clones obtained were then verified by DNA sequencing.

\section{Adipocyte differentiation and isolation of hepatocytes and adipocytes}

3T3-L1 preadipocytes were transfected with the indicated plasmids using Amaxa Nucleofector technology (Amaxa, Cologne, Germany) according to manufacturers' protocol (https://bioscience.lonza.com/lonza_bs/US/en/ document/download/21296). The differentiation of 3T3L1 cells was induced as previously described ${ }^{64}$. Briefly, 
3T3-L1 cell differentiation was induced by treatment with differentiation medium $(0.5 \mathrm{mM}$ 3-isobutyl-1methylzanthine (Sigma), $1 \mu \mathrm{M}$ dexamethasone (Sigma), and $5 \mu \mathrm{g} / \mathrm{ml}$ insulin (Roche, Switzerland)) in Dulbecco's modified Eagle's medium (DMEM) containing 10\% fetal bovine serum (FBS)) for 2 days. Culture medium was refreshed every 2 days with DMEM supplemented with $10 \% \mathrm{FBS}$ and $5 \mu \mathrm{g} / \mathrm{ml}$ insulin. At day $10-14$ of induction, fixed cells ( $4 \%$ formaldehyde, $1 \mathrm{~h}$ ) were stained with the Oil Red O dye (Chemicon). Quantification of Oil Red O staining was achieved by measuring the absorbance at $520 \mathrm{~nm}$. The isolation of hepatocytes and adipocytes was as previously described ${ }^{42,62}$.

\section{Animals and recombinant adenoviruses}

All animal experiments were approved by the Chungbuk National University, and were conducted in accordance with the approved protocols and guidelines established by the Ethics Review Committee (CBNUA966-16-02). Male C57BL/6 or p21-null mice (4-week-old) purchased from Jackson Laboratory were placed on a high-fat diet (HFD) (60\% kcal fat; D12492, Research Diets, Inc.) for 8-10 weeks. They were kept under a $12 \mathrm{~h}$ light/ dark cycle, with free access to autoclaved food and water, in specific pathogen-free (SPF) animal center. To prepare recombinant adenoviruses expressing wild-type (WT) and T55A p21, FLAG-tagged p21 plasmids (WT and T55A) were used as templates for PCR using the primers (5'GTAACTATAACGGTCATGTCAGAACCGGCTGGGG ATGTC-3' and 5'-ATTACCTCTTTCTCCTTAGGGCT TCCTCTTGGAGAAGAT-3') and the tail vein or epididymal fat pads of the HFD-fed mice were injected with adenovirus (approximately $1 \times 10^{9}$ plaque-forming units), as previously described ${ }^{9}$.

\section{Metabolic analyses}

Blood obtained from a tail vein was used to measure fed and fasted serum insulin and glucose in ad libitum-fed and $10 \mathrm{~h}$-fasted HFD-fed mice infected with adenoviruses expressing GFP, WT p21, T55A p21, or MPK38 (positive control) using an ELISA kit (Crystal Chem) and an AccuCheck glucometer (Roche), respectively ${ }^{42}$. 2-deoxyglucose uptake, ketone concentration, fatty acid oxidation rate, lipogenesis, and lipolysis were measured in HFD-fed mice infected with the indicated adenoviruses, as previously described $^{42,63,65}$.

\section{Blood metabolic parameters}

Blood samples were obtained from the abdominal aorta of $18 \mathrm{~h}$-fasted HFD-fed mice infected with adenoviruses expressing GFP, WT p21, T55A p21, or MPK38. To separate the serum, whole blood samples were centrifuged at $2500 \mathrm{rpm}$ at $4{ }^{\circ} \mathrm{C}$ for $15 \mathrm{~min}$. An ELISA kit from R\&D Systems (Joslin DERC Assay Core) was used to measure serum free fatty acid concentration. An automated serum analyzer (Hitachi 7080, Hitachi Science System Ltd, Ibaraki, Japan) was used to measure serum glucose, triglyceride, total cholesterol, HDL-cholesterol, and LDLcholesterol. A triglyceride determination kit (Sigma) was used to measure serum triglyceride concentration. Serum concentrations of TNF- $\alpha$, IL-6, IL-1 $\beta$, and MCP1 were measured as previously described ${ }^{63}$.

\section{Glucose and insulin tolerance tests (GTTs/ITTs)}

GTTs/ITTs were performed in fasted ( $\sim 18 \mathrm{~h}$ for GTTs, $\sim 6 \mathrm{~h}$ for ITTs) HFD-fed mice infected with the indicated adenoviruses. D-glucose $(2 \mathrm{~g} / \mathrm{kg}$ body mass $)$ or insulin (0.75 U/kg mass in $0.1 \%$ bovine serum albumin (BSA); human R-insulin; Eli Lilly) was administrated intraperitoneally and blood glucose was measured from tail bleeds with an Accu-Check glucometer (Roche) at indicated time points after the administration.

\section{Statistical analysis}

The data represent the means \pm SEM of at least three independent experiments, and all experiments were performed in duplicate. Statistical analysis (GraphPad Prism software, version 6.0, La Jolla, CA) was performed using one-way or two-way ANOVA with Tukey's multiple comparison test.

\section{Acknowledgements \\ This work was supported by a National Research Foundation of Korea Grant (2018R1A2A2A05018692).}

\section{Authors contributions}

H.S. performed all experiments. H.S. and H.H. analyzed data. H.H. supervised the project, designed experiments, wrote the manuscript, and acquired funding for the study.

Conflict of interest

The authors declare that they have no conflict of interest.

\section{Publisher's note \\ Springer Nature remains neutral with regard to jurisdictional claims in published maps and institutional affiliations.}

Supplementary Information accompanies this paper at (https://doi.org/ 10.1038/s41419-019-1616-z).

Received: 25 January 2019 Revised: 22 April 2019 Accepted: 29 April 2019 Published online: 16 May 2019

\footnotetext{
References

1. Beullens, M. et al. Substrate specificity and activity regulation of protein kinase MELK. J. Biol. Chem. 280, 40003-40011 (2005).

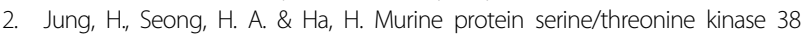
activates apoptosis signal-regulating kinase 1 via Thr 838 phosphorylation. J. Biol. Chem. 283, 34541-34553 (2008).

3. Cohen, P. The regulation of protein function by multisite phosphorylation - a 25 year update. Trends Biochem. Sci. 25, 596-601 (2000).

4. Lin, M. L., Park, J. H., Nishidate, T., Nakamura, Y. \& Katagiri, T. Involvement of maternal embryonic leucine zipper kinase (MELK) in mammary carcinogenesis
} 
through interaction with $\mathrm{BCl}-\mathrm{G}$, a pro-apoptotic member of the $\mathrm{BCl}-2$ family. Breast Cancer Res. 9, R17 (2007).

5. Seong, H. A., Kim, K. T. \& Ha, H. Enhancement of B-MYB transcriptional activity by ZPR9, a novel zinc finger protein. J. Biol. Chem. 278, 9655-9662 (2003).

6. Seong, H. A., Jung, H., Manoharan, R. \& Ha, H. PDK1 protein phosphorylation at Thr354 by murine protein serine-threonine kinase 38 contributes to negative regulation of PDK1 protein activity. J. Biol. Chem. 287, 20811-20822 (2012).

7. Seong, H. A. \& Ha, H. Murine protein serine-threonine kinase 38 activates p53 function through Ser15 phosphorylation. J. Biol. Chem. 287, 20797-20810 (2012).

8. Seong, H. A., Jung, H. \& Ha, H. Murine protein serine/threonine kinase 38 stimulates TGF-beta signaling in a kinase-dependent manner via direct phosphorylation of Smad proteins. J. Biol. Chem. 285, 30959-30970 (2010).

9. Seong, H. A., Manoharan, R. \& Ha, H. A crucial role for the phosphorylation of STRAP at Ser(188) by MPK38 in STRAP-dependent cell death through ASK1, TGF- $\beta$, p53, and PI3K/PDK1 signaling pathways. Cell Cycle 13, 3357-3374 (2014).

10. Manoharan, R., Seong, H. A. \& Ha, H. Thioredoxin inhibits MPK38-induced ASK1, TGF- $\beta$, and p53 function in a phosphorylation-dependent manner. Free Radic. Biol. Med. 63, 313-324 (2013).

11. Boulaire, J., Fotedar, A. \& Fotedar, R. The functions of the cdk-cyclin kinase inhibitor p21WAF1. Pathol. Biol. (Paris) 48, 190-202 (2000).

12. Dotto, G. P. p21(WAF1/Cip1): more than a break to the cell cycle? Biochim. Biophys. Acta 1471, M43-M56 (2000).

13. Harper, J. W., Adami, G. R., Wei, N., Keyomarsi, K. \& Elledge, S. J. The p21 Cdk interacting protein Cip1 is a potent inhibitor of G1 cyclin-dependent kinases. Cell 75, 805-816 (1993).

14. Waga, S., Hannon, G. J., Beach, D. \& Stillman, B. The p21 inhibitor of cyclin dependent kinases controls DNA replication by interaction with PCNA. Nature 369, 574-578 (1994)

15. Xiong, Y. et al. p21 is a universal inhibitor of cyclin kinases. Nature $\mathbf{3 6 6}$ 701-704 (1993).

16. Kitaura, H. et al. Reciprocal regulation via protein-protein interaction between c-Myc and p21(cip1/waf1/sdi1) in DNA replication and transcription. J. Biol. Chem. 275, 10477-10483 (2000).

17. Delavaine, L. \& La Thangue, N. B. Control of E2F activity by p21Waf1/Cip1. Oncogene 18, 5381-5392 (1999).

18. Timchenko, N. A. et al. CCAAT/enhancer binding protein alpha regulates p21 protein and hepatocyte proliferation in newborn mice. Mol. Cell. Biol. 17, 7353-7361 (1997).

19. Coqueret, O. \& Gascan, H. Functional interaction of STAT3 transcription factor with the cell cycle inhibitor p21WAF1/CIP1/SDI1. J. Biol. Chem. 275, 18794-18800 (2000)

20. Suzuki, A., Tsutomi, Y., Akahane, K, Araki, T. \& Miura, M. Resistance to Fasmediated apoptosis: activation of caspase 3 is regulated by cell cycle regulator p21WAF1 and IAP gene family ILP. Oncogene 17, 931-939 (1998).

21. Shim, J., Lee, H., Park, J., Kim, H. \& Choi, E. J. A non-enzymatic p21 protein inhibitor of stress-activated protein kinases. Nature 381, 804-806 (1996).

22. Bennett, B. L. et al. SP600125, an anthrapyrazolone inhibitor of Jun N-terminal kinase. Proc. Natl Acad. Sci. USA 98, 13681-13686 (2001).

23. Gotz, C., Wagner, P., Issinger, O. G. \& Montenarh, M. p21WAF1/CIP1 interacts with protein kinase CK2. Oncogene 13, 391-398 (1996).

24. Estanyol, J. M. et al. The protein SET regulates the inhibitory effect of p21(Cip1) on cyclin E-cyclin-dependent kinase 2 activity. J. Biol. Chem. 274, 33161-33165 (1999).

25. Taules, M. et al. Calmodulin binds to p21(Cip1) and is involved in the regulation of its nuclear localization. J. Biol. Chem. 274, 24445-24448 (1999).

26. Funk, J. O. et al. Inhibition of CDK activity and PCNA-dependent DNA replication by p21 is blocked by interaction with the HPV-16 E7 oncoprotein. Genes Dev. 11, 2090-2100 (1997).

27. Kearsey, J. M., Coates, P. J., Prescott, A. R., Warbrick, E. \& Hall, P. A. Gadd45 is a nuclear cell cycle regulated protein which interacts with p21Cip1. Oncogene 11, 1675-1683 (1995).

28. Ono, T. et al. TOK-1, a novel p21Cip1-binding protein that cooperatively enhances p21-dependent inhibitory activity toward CDK2 kinase. J. Biol. Chem. 275, 31145-31154 (2000).

29. Gorospe, M. et al. p21 (Waf1/Cip1) protects against p53-mediated apoptosis of human melanoma cells. Oncogene 14, 929-935 (1997).

30. Gorospe, M., Wang, X., Guyton, K. Z. \& Holbrook, N. J. Protective role of p21 (Waf1/Cip1) against prostaglandin A2-mediated apoptosis of human colorectal carcinoma cells. Mol. Cell. Biol. 16, 6654-6660 (1996).
31. Barboule, N. et al. Expression of p21WAF1/CIP1 is heterogeneous and unrelated to proliferation index in human ovarian carcinoma. Int. J. Cancer $\mathbf{6 3}$ 611-615 (1995).

32. Erber, R. et al. Aberrant p21(CIP1/NAF1) protein accumulation in head-andneck cancer. Int. J. Cancer 74, 383-389 (1997).

33. Esteve, $\mathbf{V}$. et al. The structural plasticity of the $\mathbf{C}$ terminus of p21Cip1 is a determinant for target protein recognition. Chembiochem 4, 863-869 (2003).

34. Inoue, N. et al. Cyclin-dependent kinase inhibitor, p21WAF1/CIP1, is involved in adipocyte differentiation and hypertrophy, linking to obesity, and insulin resistance. J. Biol. Chem. 283, 21220-21229 (2008).

35. Naaz, A. et al. Loss of cyclin-dependent kinase inhibitors produces adipocyte hyperplasia and obesity. FASEB J. 18, 1925-1927 (2004).

36. Davezac, N., Baldin, V., Blot, J., Ducommun, B. \& Tassan, J. P. Human pEg3 kinase associates with and phosphorylates CDC25B phosphatase: a potential role for pEg3 in cell cycle regulation. Oncogene 21, 7630-7641 (2002).

37. Jakobsen, S. N., Hardie, D. G., Morrice, N. \& Tornqvist, H. E. 5'-AMP-activated protein kinase phosphorylates IRS-1 on Ser-789 in mouse C2C12 myotubes in response to 5-aminoimidazole-4-carboxamide riboside. J. Biol. Chem. 276 46912-46916 (2001).

38. Child, E. S. \& Mann, D. J. The intricacies of p21 phosphorylation: protein/protein interactions, subcellular localization and stability. Cell Cycle 5, 1313-1319 (2006).

39. Kim, J. B., Wright, H. M., Wright, M. \& Spiegelman, B. M. ADD1/SREBP1 activates PPARY through the production of endogenous ligand. Proc. Natl Acad. Sci. USA 95, 4333-4337 (1998)

40. Spiegelman, B. M. PPAR-gamma: adipogenic regulator and thiazolidinedione receptor. Diabetes 47, 507-514 (1998).

41. Kubo, M., Ijichi, N., Ikeda, K., Horie-Inoue, K. \& Inoue, S. Modulation of adipogenesis-related gene expression by estrogen-related receptor $\gamma$ during adipocytic differentiation. Biochim. Biophys. Acta 1789, 71-77 (2009).

42. Seong, H. A., Manoharan, R. \& Ha, H. Coordinate Activation of RedoxDependent ASK1/TGF- $\beta$ Signaling by a Multiprotein Complex (MPK38, ASK1, SMADs, ZPR9, and TRX) Improves Glucose and Lipid Metabolism in Mice. Antioxid. Redox Signal. 24, 434-452 (2016).

43. van Diepen, J. A., Berbée, J. F., Havekes, L. M. \& Rensen, P. C. Interactions between inflammation and lipid metabolism: relevance for efficacy of antiinflammatory drugs in the treatment of atherosclerosis. Atherosclerosis 228, 306-315 (2013).

44. Sengupta, S., Peterson, T. R., Laplante, M., Oh, S. \& Sabatini, D. M. mTORC1 controls fasting-induced ketogenesis and its modulation by ageing. Nature 468, 1100-1104 (2010).

45. Kim, G. Y. et al. The stress-activated protein kinases p38a and JNK1 stabilize p21Cip1 by phosphorylation. J. Biol. Chem. 277, 29792-29802 (2002).

46. Sheaff, R. J. et al. Proteasomal turnover of p21Cip1 does not require p21Cip1 ubiquitination. Mol. Cell 5, 403-410 (2000).

47. Abbas, T. \& Dutta, A. p21 in cancer: intricate networks and multiple activities. Nat. Rev, Cancer 9, 400-414 (2009).

48. Zhang, Z. et al. MDM2 is a negative regulator of p21WAF1/CIP1, independent of p53. J. Biol. Chem. 279, 16000-16006 (2004).

49. Li, Y., Dowbenko, D. \& Lasky, L. AKT/PKB phosphorylation of p21Cip/WAF1 enhances protein stability of p21Cip/NAF1 and promotes cell survival. J. Biol. Chem. 277, 11352-11361 (2002).

50. Ro"ssig, L., Badorff, C., Holzmann, Y., Zeiher, A. M. \& Dimmeler, S. Glycogen synthase kinase-3 couples AKT-dependent signaling to the regulation of p21Cip1 degradation. J. Biol. Chem. 277, 9684-9689 (2002).

51. Zhou, B. P. et al. Cytoplasmic localization of p21Cip1MAF1 by Akt-induced phosphorylation in HER-2/neu-overexpressing cells. Nat. Cell Biol. 3, 245-252 (2001).

52. Rodriguez-Vilarrupla, A. et al. Binding of calmodulin to the carboxy-terminal region of p21 induces nuclear accumulation via inhibition of protein kinase $C$ mediated phosphorylation of Ser153. Mol. Cell. Biol. 25, 7364-7374 (2005).

53. Mercer, S. E. et al. Mirk/Dyrk1B mediates sunvival during the differentiation of C2C12 myoblasts. J. Biol. Chem. 280, 25788-25801 (2005).

54. Jarviluoma, A. et al. Phosphorylation of the cyclin-dependent kinase inhibitor p21Cip1 on serine 130 is essential for viral cyclin-mediated bypass of a p21Cip1-imposed G1 arrest. Mol. Cell. Biol. 26, 2430-2440 (2006).

55. MacDougald, O. A. \& Mandrup, S. Adipogenesis: forces that tip the scales. Trends Endocrinol. Metab. 13, 5-11 (2002).

56. Farmer, S. R. Transcriptional control of adipocyte formation. Cell Metab. 4 263-273 (2006). 
57. Rosen, E. D. et al. PPAR gamma is required for the differentiation of adipose tissue in vivo and in vitro. Mol. Cell 4, 611-617 (1999).

58. Barak, Y. et al. PPAR gamma is required for placental, cardiac, and adipose tissue development. Mol. Cell 4, 585-595 (1999).

59. Wu, Z. et al. Cross-regulation of C/EBP alpha and PPAR gamma controls the transcriptional pathway of adipogenesis and insulin sensitivity. Mol. Cell $\mathbf{3}$ 151-158 (1999).

60. Kim, S. W., Her, S. J., Kim, S. Y. \& Shin, C. S. Ectopic overexpression of adipogenic transcription factors induces transdifferentiation of MC3T3-E1 osteoblasts. Biochem. Biophys. Res. Commun. 327, 811-819 (2005)

61. Lefterova, M. I., Haakonsson, A. K., Lazar, M. A. \& Mandrup, S. PPARy and the global map of adipogenesis and beyond. Trends Endocrinol. Metab. 25, 293-302 (2014).
62. Seong, H. A., Manoharan, R. \& Ha, H. Zinc finger protein ZPR9 functions as an activator of AMPK-related serine/threonine kinase MPK38/MELK involved in ASK1/TGF-B/p53 signaling pathways. Sci. Rep. 7, 42502 (2017).

63. Seong, H. A., Manoharan, R. \& Ha, H. Smad proteins differentially regulate obesity-induced glucose and lipid abnormalities and inflammation via class-specific control of AMPK-related kinase MPK38/MELK activity. Cell Death Dis. 9, 471 (2018).

64. Park, B. H. et al. Mammalian ste20-like kinase and SAV1 promote 3T3-L1 adipocyte differentiation by activation of PPARY. PLOS ONE 7, e30983 (2012).

65. Chang, P. Y. et al. Overexpression of hexokinase II in transgenic mice: evidence that increased phosphorylation augments muscle glucose uptake. J. Biol. Chem. 271, 14834-14839 (1996). 